\title{
The evaluation of a typing scheme for coagulase- negative staphylococci suitable for epidemiological studies
}

\author{
H. A. LUdLAM, W. C. NOBLE, R. R. MARPLES* and I. PHILLIPS \\ Division of Microbiology and Institute of Dermatology, UMDS, St Thomas' Hospital, London SE1 7EH and \\ "Staphylococcus Reference Unit, Central Public Health Laboratory, Colindale, London NW9 5 HT
}

\begin{abstract}
Summary. A typing scheme was devised for an epidemiological study of infection with coagulase-negative staphylococci in patients undergoing continuous ambulatory peritoneal dialysis (CAPD). The scheme was constructed in four stages suitable for the screening of large numbers of isolates: antibiogram, biotype, phage type, and plasmid profile. The discrimination and reproducibility of the scheme was established by the examination of 50 isolates from 33 consecutive episodes of peritonitis affecting 18 patients. The discrimination of the scheme was $76 \%$, with a reproducibility of $86 \%$. Indistinguishable strains occurred in individual patients only, demonstrating that no cross-infection between patients occurred during the 10-month period of collection of strains, and suggesting that the discriminating power of the scheme was, in fact, much higher. The antibiogram, selected as the first stage of the scheme because it was the simplest and cheapest test, proved to be the most discriminatory stage, providing $66 \%$ of the final discriminatory power.
\end{abstract}

\section{Introduction}

The commonest infecting organisms in continuous ambulatory peritoneal dialysis (CAPD) peritonitis are coagulase-negative staphylococci, yet very little is known of the origin of these organisms. The patient's own skin flora has been assumed to be the source of each of these infections. We wished to confirm this by an epidemiological study in which infecting strains were to be sought amongst a large number of coagulase-negative staphylococci collected from the skin of the CAPD patients. First, it was necessary to develop and test a typing scheme suitable for such a study. The methods described for the typing of coagulase-negative staphylococci include antimicrobial susceptibility patterns (antibiogram) (Richardson and Marples, 1982), biochemical characterisation (biotyping) (Holt, 1969), bacteriophage susceptibility patterns (conventional phage typing) (de Saxe et al., 1981) and reverse phage typing (de Saxe and Notley, 1978), serological typing (serotyping) (Aasen and Oeding, 1971), bacteriolytic activity patterns (lyogroup) (Varaldo et al., 1980), molecular analysis of plasmids (plasmid profiles) (Parisi and Hecht, 1980), restriction enzyme analysis and DNA-DNA hybridisation (Par-

Received 23 Aug. 1988; revised version accepted 5 Apr. 1989. isi, 1985), and synthesis of a mucoid substance (slime production) (Christensen et al., 1983). No single method has proved satisfactory and combinations of these tests have been required to discriminate between unrelated strains (Christensen et al., 1983; Parisi and Hamory, 1986; Eisenberg et al., 1987; Hartstein et al., 1987). We selected a combination of four well established methods for our typing scheme. The scheme was constructed as follows: antibiogram with limited biotype (first stage), biotype by API-Staph profile (second stage), phage typing (third stage), and plasmid profile (fourth stage).

We applied the typing scheme to 50 consecutive isolates of coagulase-negative staphylococci from patients with CAPD peritonitis to establish its discrimination and reproducibility.

\section{Materials and methods}

\section{Source of strains}

Fifty strains, characterised as coagulase-negative staphylococci by the diagnostic laboratory, were derived from the peritoneal fluid in 33 consecutive episodes of CAPD peritonitis involving 18 patients at St Thomas' Hospital. Multiple isolates were obtained in 17 episodes and 8 patients experienced more than one episode of 
peritonitis. The strains were stored at $-70^{\circ} \mathrm{C}$ in glycerol (0201/0042; Kirby Warrick Pharmaceuticals Ltd, Suffolk) $7 \%$ in Bacto Nutrient Broth (0003; Difco Ltd, East Molesey, Scotland) and recovered for this study by subculture to blood agar (Columbia Agar base, CM331; Oxoid Ltd, Basingstoke, Hampshire), with 7\% horse blood (102-00001 H; Bio-Cult Diagnostics Ltd, Paisley, Scotland), incubated at $37^{\circ} \mathrm{C}$ for $18 \mathrm{~h}$.

\section{Characters investigated}

\section{First stage: Antibiogram with limited biotype}

Plating procedure. One colony was transferred to one quarter of the surface of a Mannitol Salt Agar plate (MSA agar, CM85; Oxoid). One quarter of a blood agar plate (for methicillin sensitivity testing) was inoculated from the mannitol salt agar plate. Two lysed blood agar sensitivity plates (Oxoid DST medium, CM261) with 6\% horse blood lysed by white saponin $(44092$; BDH Chemicals Ltd, Poole) and a Cysteine Lactose Electrolyte Deficient purity plate (CLED Agar, 152-1240; Gibco Europe Ltd, Paisley, Scotland) were inoculated from the blood agar plate. A second colony was taken to construct an identical series of duplicate plates (series B) for the reproducibility study. These were assigned a separate code unknown to the investigators. Further purity plates were prepared from the methicillin blood agar plates of series A and series B for supplementary typing by biotyping, phage typing and plasmid profile.

Antibiotic sensitivities. Isolates were tested for sensitivity by the comparative disk-diffusion method (Brown and Blowers, 1978) to the following antibiotics: penicillin $5 \mathrm{iu}$, methicillin $10 \mu \mathrm{g}$, gentamicin $10 \mu \mathrm{g}$, amikacin $30 \mu \mathrm{g}$, streptomycin $25 \mu \mathrm{g}$, neomycin $30 \mu \mathrm{g}$, vancomycin $30 \mu \mathrm{g}$, tetracycline $50 \mu \mathrm{g}$, minocycline $30 \mu \mathrm{g}$, erythromycin $5 \mu \mathrm{g}$, clindamycin $2 \mu \mathrm{g}$, fusidic acid $10 \mu \mathrm{g}$, rifampicin $2 \mu \mathrm{g}$, trimethoprim $2.5 \mu \mathrm{g}$, chloramphenicol $50 \mu \mathrm{g}$, novobiocin $5 \mu \mathrm{g}$, bacitracin 0.04 units and furazolidone $100 \mu \mathrm{g}$. The definitions of sensitivity were for taxonomic and epidemiological, rather than therapeutic, purposes.

Sensitivity plates were read after incubation for $24 \mathrm{~h}$ at $37^{\circ} \mathrm{C}$ (lysed blood agar plates) or $30^{\circ} \mathrm{C}$ (methicillin blood agar plates). Isolates were recorded as either sensitive or resistant to the antibiotics (isolates with zone sizes indicating intermediate sensitivity were regarded as resistant). For bacitracin, used for identification of micrococci, organisms displaying any zone of inhibition were recorded as sensitive (Falk and Guering, 1983).

Mannitol reaction. The salt mannitol plate was examined after incubation for 24 and $48 \mathrm{~h}$. Yellowing of the medium due to mannitol fermentation was recorded as a positive reaction.

Lactose reaction. The CLED plate was examined after incubation for 24 and $48 \mathrm{~h}$. Yellowing of the medium, due to fermentation, was recorded as a positive reaction.

Haemolysis. The blood agar plate was examined for haemolysis after incubation for $24 \mathrm{~h}$ at $30^{\circ} \mathrm{C}$. Organisms displaying a rim of haemolysis around the area of growth were considered haemolytic.
The 50 isolates and their duplicates were examined in one batch, for all characters investigated.

\section{Second stage : Biotype}

The isolates were typed by their biochemical profiles obtained with API-Staph strips (API Products Ltd, Basingstoke, Hampshire). The strips were inoculated and read after incubation for $24 \mathrm{~h}$, according to the manufacturer's instructions. The final reaction in the Analytical Profile, the lysostaphin test, included to differentiate micrococci and staphylococci, was not performed, but was deduced from the bacitracin and furazolidone sensitivities. The isolates were considered to be staphylococci if they were resistant to bacitracin and sensitive to furazolidone (Falk and Guering, 1983; Baker, 1984).

The 50 isolates and their duplicates were examined in one batch.

\section{Third stage: Phage type}

Phage typing was performed at the Staphylococcus Reference Unit, Colindale, London, according to the scheme developed by Dean et al. (1973). The results were interpreted as described by de Saxe et al. (1981): isolates showing more than 50 plaques at $100 \times$ Routine Test Dilution (reported as ++ and $C L$ ) were considered typable. Discrimination between strains was based on one Strong Difference, defined as a strong reaction $(++$ and CL) compared with a negative reaction (Not Typable, NT). Weak reactions $(+, \pm)$ and inhibition reactions $(0)$ were considered to be an intermediate group providing no useful information.

The 50 isolates and their duplicates were tested in one batch.

\section{Fourth stage: Plasmid profile}

Plasmid profiles were prepared and interpreted by the method of Degener et al. (1987). Isolates were considered to be indistinguishable if they possessed plasmids of identical mobility (but see Discussion).

The 50 isolates with their duplicates could not be examined together and were, therefore, examined in six batches.

\section{Results}

\section{First stage: Antibiogram with limited biotype}

Antibiogram. Discrimination: The 50 isolates demonstrated 25 distinct patterns of antibiotic resistance. The discriminatory power of this test was therefore $50 \%$. Reproducibility: The results of the antibiogram for series B were different for one pair only ( $98 \%$ reproducibility). Retesting demonstrated that the discrepant result was due to observer error in the interpretation of zone size for an isolate with borderline sensitivity. 
Limited biotype. Eight isolates fermented mannitol at $24 \mathrm{~h}$ and a further one gave a positive result at $48 \mathrm{~h}$. Forty six isolates fermented lactose at $24 \mathrm{~h}$ and a further one gave a positive result at $48 \mathrm{~h}$. Five isolates were haemolytic.

Performance of the first stage. The results of mannitol fermentation and haemolysis each contributed a further two distinguishable groups to the 25 obtained with the antibiogram alone, producing a final result of 29 distinct groups (58\% discrimination), and $98 \%$ reproducibility.

\section{Second stage: Biotype}

Biotyping by the API-Staph system yielded 13 groups with distinct API-Staph profiles $(26 \%$ discrimination). The profiles for series B differed in one pair only ( $98 \%$ reproducibility).

Forty-one isolates were identified as Staphylococcus epidermidis, one of the seven profiles for this species (6606113) accounting for 30 isolates. Four isolates were identified as $S$. simulans, two each as $S$. hominis and $S$. haemolyticus and one isolate was identified as a micrococcus.

The discrepant result was due to an incorrect profile in one strip, not reproduced on retesting. This anomalous result was presumably due to a faulty strip or to a technical error.

\section{Third stage: Phage type}

Thirteen isolates demonstrated strong lysis by at least one phage, corresponding to $26 \%$ typability.

The discrimination of the technique was $14 \%$ (seven distinct groups) when isolates were distinguished by two strong differences, increasing to $18 \%$ (nine groups) when isolates were distinguished by one strong difference. As the reproducibility of the technique was $100 \%$ (no discrepant results on re-testing) discrimination at the level of one strong difference was selected for the typing scheme.

\section{Fourth stage: Plasmid profile}

The 50 isolates showed 24 distinct plasmid profiles, corresponding to a discrimination of $48 \%$. Five pairs showed discrepant results on re-testing, corresponding to a reproducibility of $90 \%$.

Eight of the 24 profiles contained multiple isolates (to a maximum of eight). Four isolates contained no plasmids, and one further isolate which failed to type was the micrococcus.

\section{Overall performance of the typing scheme}

Discrimination. The 50 isolates were divided into 38 distinct groups (76\% discrimination). Five groups contained more than one isolate. In each of these groups the isolates were derived from episodes of peritonitis affecting one patient only.

Reproducibility. The overall reproducibility of the scheme was $86 \%$.

\section{Discussion}

The aim of this study was to develop a practical typing scheme that could be applied to an epidemiological study expected to generate large numbers of skin isolates for testing for similarity to an infecting (peritonitis) strain. The typing scheme was constructed in four consecutive stages of increasing cost and complexity. It was hoped that the first stage, the antibiogram with limited biotype (the simplest and cheapest test), would act as an efficient screen so that the majority of skin strains could be discarded and relatively few isolates would have to be examined by the more expensive and demanding tests. Despite the poor reputation that the antibiogram has acquired as an epidemiological tool (Marples, 1986), we found the antibiogram not only reproducible but also highly discriminatory, and an excellent primary screen (providing $66 \%$ of the scheme's overall discriminatory power). We have found it equally effective in a study of the epidemiology of infection in individual CAPD patients (Ludlam et al., 1989). However, the microbial flora of our CAPD patients is frequently exposed to antibiotics and it cannot be assumed that the antibiogram would be equally effective for all patient groups. In 1986 CAPD patients at our unit received treatment for an average of two episodes of peritonitis, and half were prescribed antibiotics for Tenckhoff exit-site infections.

Species determination would normally be the first step in a typing scheme for the characterisation of individual isolates. However, our scheme was designed for epidemiological purposes and screening by the API-Staph biotype was relegated to the secondary stage because not only is it more expensive but it was expected to be poorly discriminatory (Parisi and Hamory, 1986; Hartstein et al., 1987), as confirmed in the present study. Furthermore, its use as a primary screen in prospective epidemiological studies of infection with coagulasenegative staphylococci has almost certainly resulted in the erroneous elimination of strains that would be found dissimilar by other methods of typing, such as the antibiogram (Eisenberg et al., 1987). 
Although the discriminatory power of biotyping by API-Staph profile was $26 \%$, it made no final contribution to the discrimination of our scheme in view of the duplication of its results by the later stages, phage typing and plasmid profile. However, it is readily available and easy to perform and, in combination with the extended antibiogram, it achieved $87 \%$ of the scheme's final discriminatory power.

Although formal rules for the interpretation of the results of phage typing of coagulase-negative staphylococci have yet to be established, the perfect reproducibility demonstrated in this study suggests that discrimination between strains at the level of one Strong Difference is acceptable when the test strains are examined in one batch. Other workers have reported similar findings (Verhoef et al., 1972). However, even at this level, phage-typing was the least discriminatory test ( $18 \%$ discrimination), and species other than $S$. epidermidis were untypable; this is a recognised shortcoming (Dean et al., 1973; de Saxe and Notley, 1978).

Plasmid typing was only slightly less discriminatory than the antibiogram, and did not duplicate the latter's results, many strains possessing plasmids of unknown function in addition to plasmids coding for antibiotic resistance. Indeed, the combination of the antibiogram and the plasmid profile achieved $95 \%$ of the final discriminatory power of the scheme. We have assumed that plasmids of similar electrophoretic mobility are identical, but this may not always be the case. DNA-DNA hybridisation studies can resolve the issue (Parisi, 1985), but this is technically demanding.

Analysis of the results shows that the antibiogram with limited biotype successfully separated 29 of the 50 isolates. Biotyping or phage typing of the remaining 21 isolates yielded a further four types only, whilst plasmid typing of the 21 isolates yielded a further seven types. These results suggest that if limited resources were to dictate a shortening of the typing scheme, the choice would lie between antibiogram with biotyping and phage typing, or antibiogram with plasmid-profile determination, both approaches achieving $95 \%$ of the final discriminatory power of the scheme. Other workers have reported similar findings with blood-culture isolates (Hartstein et al., 1987).

A novel typing method employing automated pattern matching of radiolabelled proteins separated by gel electrophoresis has recently been applied to the typing of coagulase-negative staphylococci and found to be equivalent in discrimination to an antibiogram with API-profile biotype (Stephenson and Tabaqchali, 1986). Analysis of the pattern of antigenic bands of intracellular proteins as demonstrated by rabbit hyperimmune globulin, after gel electrophoresis (immunoblot fingerprinting) is a further specialised technique currently undergoing evaluation. This typing method has been found to be of equivalent power to a combination of short antibiogram, API-profile and phage typing (Burnie et al., 1988). These techniques are complex and unlikely to prove suitable for epidemiological investigations that involve the examination of very large numbers of coagulasenegative staphylococci derived from skin surveys.

The typing scheme as a whole generated 38 distinguishable groups, achieving a satisfactory $76 \%$ final discrimination. Six groups contained more than one isolate, but all isolates in each of these groups were derived from episodes involving the same patient. Thus the scheme demonstrated that during the 10-month period of collection of isolates there was no episode of cross-infection between patients. This finding also suggests that these indistinguishable isolates were indeed identical, being derived from relapsing infections, and casts doubt on the value of the addition of further discriminatory tests to the scheme.

Great care was taken to ensure reproducibility. All isolates were examined together for the extended antibiogram, biotype and phage type, minimising variabilities in bench technique, media and reagents, period of incubation and interpretation of results. A source of error that was anticipated, but not observed in this study, was variability in the antibiogram due to plasmid loss. Nevertheless, it is clearly important to establish the variability of an infecting strain when searching for similar skin strains in epidemiological studies. The finding that genes controlling carbohydrate fermentation and phage adsorption in coagulase-negative staphylococci may also be located on a plasmid suggests that this also applies to the biotype and phage type (Schaefler, 1972). Reproducibility for these tests was excellent, but this was not so for plasmidprofile determination, due to the impossibility of examining all 100 isolates together. This is a further reason, in addition to expense, for reserving the plasmid profile to a late stage in any typing scheme to be applied to the epidemiology of coagulasenegative staphylococci.

We have not taken into account the fact that, for 'in-house' studies, a degree of judgment, or more subjective approach, would be taken with isolates thought likely to be the same, e.g., sequential recoveries of organisms from a single source over a short period. Such an approach might increase the value of plasmid profiles and of the minor reactions 
encountered in phage typing. The results presented here represent the most stringent analysis of our findings.

We have established a practical typing scheme for coagulase-negative staphylococci, suitable for epidemiological studies, that is both discriminatory and reproducible. The first stage, based on the antibiogram, was not only the cheapest and easiest to perform, but also the most discriminatory, so that the majority of skin strains dissimilar to any

\section{REFERENCES}

Aasen J, Oeding P 1971 Antigenic studies on Staphylococcus epidermidis. Acta Pathologica, Microbiologica et Immunologica Scandinavica Section B, 79: 827-834.

Baker J S 1984 Comparison of various methods for differentiation of staphylococci and micrococci. Journal of Clinical Microbiology 19: 875-879.

Brown D, Blowers R 1978 Disc methods of sensitivity testing and other semiquantitative methods. In: Reeves D S et al. (eds) Laboratory methods in antimicrobial chemotherapy. Churchill Livingstone, Edinburgh, pp 8-30.

Burnie J P, Lee W, Matthews R C, Bayston R 1988 Immunoblot fingerprinting of coagulase negative staphylococci. Journal of Clinical Pathology 41 : 103-107.

Christensen G D, Parisi J T, Bisno A L, Simpson W A, Beachey E H 1983 Characterization of clinically significant strains of coagulase-negative staphylococci. Journal of Clinical Microbiology 18: 258-269.

de Saxe M J, Crees-Morris J A, Marples R R, Richardson J F 1981 Evaluation of current phage-typing systems for coagulase-negative staphylococci. Zentralblatt fur Bakteriologie, Mikrobiologie und Hygiene Suppl 10: 197-204.

de Saxe M J, Notley C M 1978 Experiences with the typing of coagulase-negative staphylococci and micrococci. Zentralblatt fur Bakteriologie, Mikrobiologie, Parasitenkunde, Infektionskrankheiten und Hygiene Abteilung I, Originale A, 241 : 46-59.

Dean B A, Williams R E O, Hall F, Corse J 1973 Phage typing of coagulase-negative staphylococci and micrococci. Journal of Hygiene 71 : 261-270.

Degener J E, Naidoo J L, Noble W C, Phillips I, Marples R R 1987 Carriage of gentamicin-resistant coagulase-negative staphylococci in patients on continuous ambulatory peritoneal dialysis. Journal of Antimicrobial Chemotherapy 19: 505-512.

Eisenberg E S, Ambalu M, Szylagi G, Aning V, Soeiro R 1987 Colonisation of skin and development of peritonitis due to coagulase-negative staphylococci in patients undergoing peritoneal dialysis. Journal of Infectious Diseases 156: 478482.

Falk D, Guering S J 1983 Differentiation of Staphylococcus and infecting strain could be discarded at this stage, sparing the more costly and time-consuming later stages.

We are grateful to Mary Rahman for preparing the plasmid profiles, to Dr J. E. Degener for his helpful advice, and to Drs N. F. Jones, A. J. Wing and P. J. Hilton for permission to study their patients. H. A. Ludlam was supported by a grant from Research Endowments, St Thomas' Hospital. Mary Rahman was supported by a grant from the National Kidney Research Fund.

Micrococcus spp. with the Taxo A bacitracin disk. Journal of Clinical Microbiology 18: 719-721.

Hartstein A I, Valvano M A, Morthland V H, Fuchs P C, Potter S A, Crosa J H 1987 Antimicrobic susceptibility and plasmid profile analysis as identity tests for multiple blood isolates of coagulase-negative staphylococci. Journal of Clinical Microbiology 25 : 589-593.

Holt R 1969 The classification of staphylococci from colonized ventriculo-atrial shunts. Journal of Clinical Pathology 22: 475-482.

Ludlam H A, Noble W C, Marples R R, Bayston R, Phillips I 1989 The epidemiology of peritonitis caused by coagulasenegative staphylococci in continuous ambulatory peritoneal dialysis. Journal of Medical Microbiology 30: 167-174.

Marples R R 1986 Laboratory assessment in the epidemiology of infections caused by coagulase-negative staphylococci. Journal of Medical Microbiology 22 : 285-286.

Parisi J T 1985 Coagulase-negative staphylococci and the epidemiological typing of Staphylococcus epidermidis. Microbiological Reviews 49: 126-139.

Parisi J T, Hamory B H 1986 Simplified method for the isolation, identification, and characterization of Staphylococcus epidermidis in epidemiologic studies. Diagnostic Microbiology and Infectious Diseases 4: 29-35.

Parisi J T, Hecht D W 1980 Plasmid profiles in epidemiologic studies of infections by Staphylococcus epidermidis. Journal of Infectious Diseases 141: 637-643.

Richardson J F, Marples R R 1982 Changing resistance to antimicrobial drugs, and resistance typing in clinically significant strains of Staphylococcus epidermidis. Journal of Medical Microbiology 15: 475-484

Schaefler S 1972 Polyfunctional penicillinase plasmid in Staphylococcus epidermidis: bacteriophage restriction and modification mutants. Journal of Bacteriology 112: 697-706.

Stephenson J R, Tabaqchali S 1986 New method for typing coagulase negative staphylococci. Journal of Clinical Pathology 39: 1271-1275.

Varaldo P E, Grazi G, Soro O, Cisani G, Satta G 1980 Simplified lyogroup System, a new method for routine identification of staphylococci: description and comparison with three other methods. Journal of Clinical Microbiology 12: 63-68.

Verhoef J, Van Boven C P A, Winkler K C 1972 Phage-typing of coagulase-negative staphylococci. Journal of Medical Microbiology 5: 9-19. 